\title{
Multilayer coatings for Bloch surface wave optical biosensors
}

\author{
Peter Munzert ${ }^{1}$, Norbert Danz ${ }^{1}$, Francesco Michelotti ${ }^{2}$, \\ ${ }^{1}$ Fraunhofer Institute for Applied Optics and Precision Engineering, Jena, Germany \\ ${ }^{2}$ SAPIENZA University of Rome, Basic and Applied Science for Engineering \\ Department (SBAI), Rome, Italy
}

\begin{abstract}
Sensors using surface plasmon resonance (SPR) are established as the method of choice in label-free optical biosensing. Their sensitivity for small refractive index changes at the surface originates from the enhanced evanescent field at the surface of a thin metal layer. However, the small number of well-suited metals $(\mathrm{Ag}, \mathrm{Au})$ with fixed optical constants limits a further refinement of the SPR performance in terms of dispersion and resonance width. An alternative can be found in Bloch Surface Waves (BSW) sustained at specially designed dielectric multilayer stacks with low absorption losses. Due to the low losses an enormous narrowing of the resonance is obtained, promising the reduction of the detection limit for such a label-free sensor. Furthermore, fluorescence enhancement caused by near field effects can also be exploited and, by engineering the BSW dispersion, both detection schemes can be combined. This paper illustrates the basic principles of BSW generation by designing thin dielectric multilayer stacks and the vacuum deposition of these coatings on disposable polymer biochips. In addition, the measurement and analysis of the Bloch resonance shift caused by the interaction of biomolecules at extremely small concentrations is presented.
\end{abstract}

\section{Introduction}

The increasing demand for early detection of diseases drives the efforts to develop increasingly sensitive techniques to detect biomarkers in extremely low concentrations. For this, a standard clinical verification method is ELISA [1]. Here a color change reaction of enzyme labeled antibodies takes place whichplace, which enables the detection of extremely low concentrations. However, ELISA is rather slow and the reaction kinetics cannot be tracked. An alternative optical biosensing method is surface plasmon resonance (SPR) $[2,3]$ that has evolved to be the standard approach among various label-free optical methods [4]. However, the resonance exploited in plasmon based sensing is governed by the optical properties of the metal film used. The metals of choice are Gold or Silver; usually only Gold is used for practical reasons. This results in missing flexibility in operation wavelength and angle of the resonance peak. Furthermore, the resonance width is determined by the metal losses and cannot be narrowed to lower the limit of detection. A possibility to overcome these drawbacks in optical sensor design is to utilize surface modes at specially tailored dielectric multilayer stacks $[5,6]$. In such case, called Bloch surface wave, the maximum field intensity is close to the surface of the multilayer stack and an evanescent field extends into the superstrate and is exploited for sensing [7-9]. As appropriate dielectric materials for these multilayers usually exhibit much less absorption than metal films, enormous field enhancement factors associated with potentially very narrow resonances have been observed $[10,11]$. Advantages of this Bloch surface wave approach in contrast to SPR are that the BSW dispersion can be almost arbitrarily tuned by the stack design and the layer materials used. This means that BSW can operate at any wavelength and the resonance angle can also be adjusted to the sensitivity maximum [12]. Another Other benefits of the Bloch wave approach is-come from the fact; that the increased surface field enhancement and the absence of the metal layer's quenching can be exploited for fluorescence enhancement of dye molecules at the sensor surface. Compared to conventional fluorescence analysis where Since-the emission is spread over the whole half-space for conventional fluorescence analysis-and only a small amount of the light reaches the detector, the BSW approach allows to concentrate the emission into a small angular range. The emission is not attenuated by metal losses and fluorescence excitation is significantly boosted when performed by the evanescent field of a BSW.

Therefore, the new approach is to combine label-free [13] and fluorescence signal detection in only one sensor element [14] that uses such a Bloch multilayer stack. As displayed in Figure 1, the Kretschmann-Raether configuration with a glass prism [15] is applied but instead of the metal film, which is commonly used for SPR, a dielectric multilayer stack has to be deposited. By illumination with TE polarized light under total internal reflection conditions, a sharp drop in reflectivity appears at the resonance angle when the in-plane propagation constant of the illumination matches that of the surface wave. The reflected light for label-free detection and the spontaneously emitted fluorescence are then observed in the same angular range and can be recorded with the same detector [13]. 


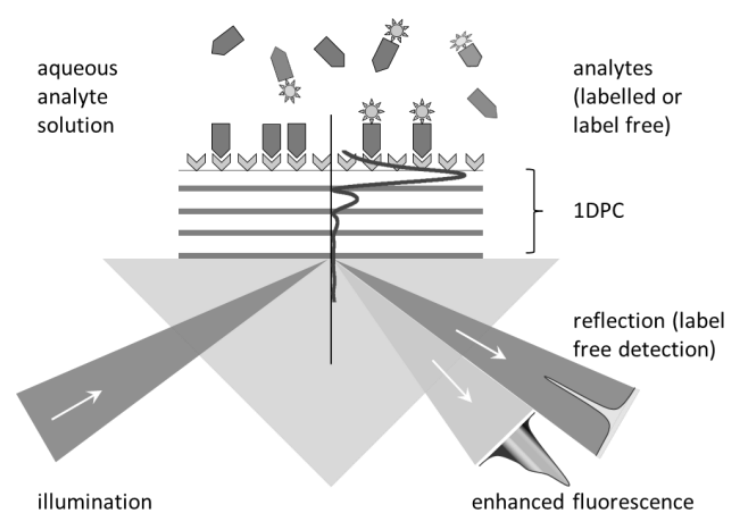

Figure 1. Scheme of a Bloch surface wave optical biosensor for label-free and fluorescence detection

With a corresponding sensor chip as the core element, an analytical biosensing instrument for the measurement of small concentrations of a typical cancer biomarker was developed.

\section{Multilayer design for BSW}

As the design base for the Bloch stacks a periodic high $(\mathrm{H}) /$ low $(\mathrm{L})$ reflector was applied as the light inside the stack has to be optimally reflected to achieve the resonant mode with the light that is totally reflected at the upper boundary. Considering such HL stacks, a common thin film calculation software can indeed calculate the resonance in reflection from a given stack but does not have an efficient reverse engineering option to optimize the resonance properties. Thus, all resonance properties were calculated in forward direction for a variation of layer thickness combinations and the optimal ranges were taken from these plots.

By the design and the material properties of the multilayer stack, the performance of the resonance peak in terms of dispersion as well as resonance width, depth and shift, can be adapted. Therefore, one of the main determining factors is the absorption losses of the layer materials. As shown in Figure 2, extremely narrow resonances can be achieved with a material absorption of $3 \times 10^{-6}$. Increasing such losses yields considerably broadened resonances.
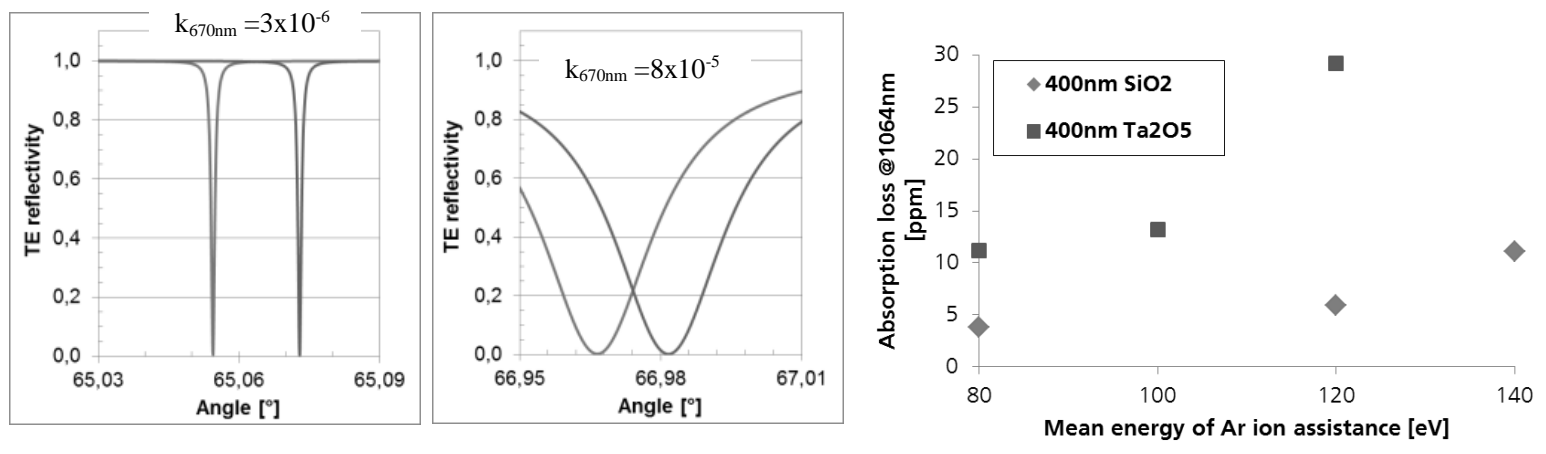

Figure 2. Optimized reflection resonances for different absorption losses of the dielectric materials in periodic BSW stacks (left: $\mathrm{k}_{670 \mathrm{~nm}}=3 \times 10^{-6}$, middle: $\mathrm{k}_{670 \mathrm{~nm}}=8 \times 10^{-5}$ ) are shown for two different refractive indices of the superstrate to illustrate the resonance shift. Experimentally determined losses @ $1064 \mathrm{~nm}$ for $\mathrm{SiO}_{2}$ and $\mathrm{Ta}_{2} \mathrm{O}_{5}$ single layers in dependence of the Ar ion assistance energy while deposition (right diagram).

Absorption losses for $\mathrm{SiO}_{2}$ and $\mathrm{Ta}_{2} \mathrm{O}_{5}$ single layers were determined by laser calorimetry measurements at $1064 \mathrm{~nm}$ wavelength. It was possible to minimize the material losses by reducing the energy for the Ar ion assistance that is applied for the vacuum evaporation process. Figure 2 shows that the absorption of a $400 \mathrm{~nm}$ thick $\mathrm{SiO}_{2}$ layer could be reduced to $3 \mathrm{ppm}$ and for $\mathrm{Ta}_{2} \mathrm{O}_{5}$ down to $12 \mathrm{ppm}$. These values correspond to $\mathrm{k}_{\mathrm{SiO} 2} \sim 2 \times 10^{-6}$ and $\mathrm{k}_{\mathrm{Ta} 2 \mathrm{O} 5} \sim 1 \times 10^{-5}$, respectively, if an interpolation to the aspired operation wavelength for the analytical instrument of $670 \mathrm{~nm}$ is performed. The number of layer periods in the stack determines the resonance width and depth depending on the material properties. In our case, two periods yield a too wide and shallow resonance that becomes too narrow for 
more than 4 periods. Thus, the optimal number of periods is between three and four, resulting in total thicknesses of these BSW stacks ranging from $1 \mu \mathrm{m}$ to $2 \mu \mathrm{m}$.

For the final stack, a non-periodic design was chosen due to a sensitivity for refractive index changes that is three times higher compared to a periodic one. In order to reduce the total thickness of the coating to $1 \mu \mathrm{m}$ and increase the resonance depth, a $20 \mathrm{~nm}$ thin $\mathrm{TiO}_{2}$ high index layer was set on top of two periods $\mathrm{SiO}_{2} / \mathrm{Ta}_{2} \mathrm{O}_{5}$ (Figure 3). It is clear that the resonance width was broadened by the elevated losses but limits the propagation length of the surface mode to scales $<1 \mathrm{~mm}$, that are compatible with spot sizes in biochemical sensors. The uppermost $20 \mathrm{~nm}$ thin $\mathrm{SiO}_{2}$ layer was solely introduced to promote silane coupling chemistry and not for optical reasons.

\section{Multilayer stack deposition on plastic sensor chips}

The basic idea was to use an injection molded polymer chip where all optical coupling elements are already integrated. In contrast to state-of-the-art glass chips, polymer chips can be manufactured very easily and cost effectively in large quantities by the injection molding technology, so the polymer chip can be used as a disposable. Furthermore, the adapter element for microfluidics that enables the analyte solution to reach the chip surface can be adjusted on the chip as a separate click-in element prepared by injection molding as well (see Figure 3).
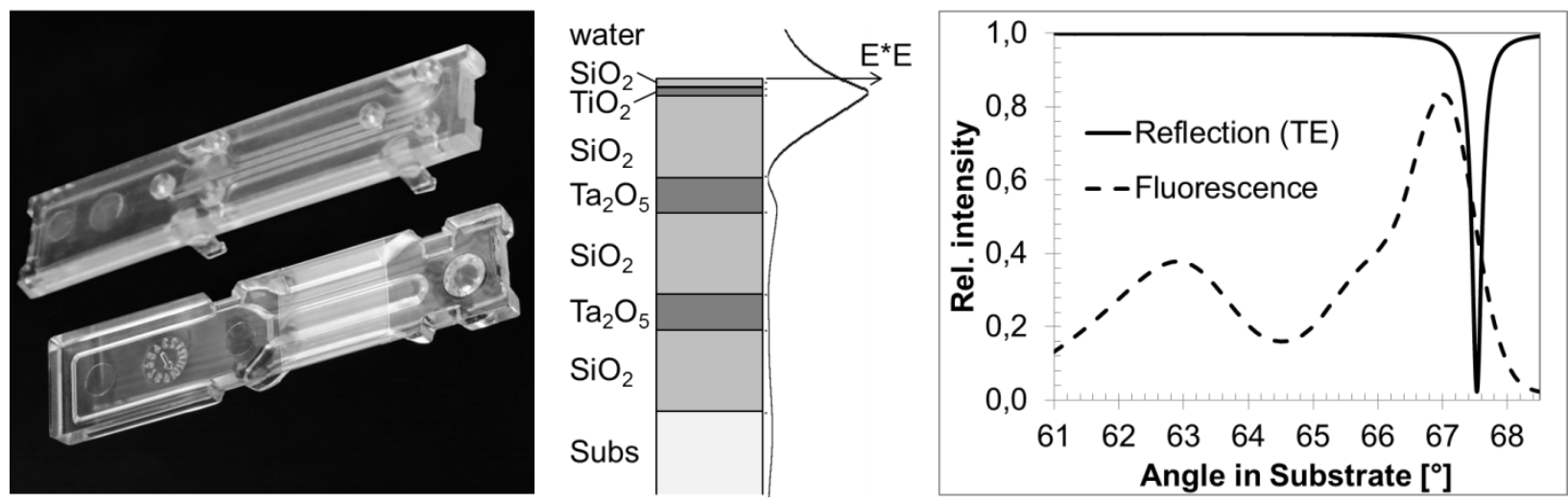

Figure 3. Polymer chip (coated with a BSW multilayer) with adapter plate for the microfluidic system (left), optimized stack design with distribution of energy density (middle), and simulated angular spectra observed in labelfree mode at $670 \mathrm{~nm}$ wavelength or in fluorescence mode for the dye DyLight650.

It was therefore necessary to deposit a micrometer thick dielectric multilayer stack on a polymer substrate. As coating technology we chose plasma ion assisted vacuum evaporation (PIAD) with a Bühler/Leybold Optics APS 904 box coater. This technique allows the deposition of dense coatings without substrate heating, so in general it is suitable for coating plastics. The film densification is performed by the APS plasma ion source, which emits high energetic Ar-ions during the evaporation process in order to densify the growing film. By a variation of the ion energy the film densification can easily be adjusted, for example to balance intrinsic film stress. Ion energies of approximately $100 \mathrm{eV}$ were applied for $\mathrm{SiO}_{2}$ with a deposition rate $0.5 \mathrm{~nm} / \mathrm{s}$ and for $\mathrm{Ta}_{2} \mathrm{O}_{5}$ with $0.4 \mathrm{~nm} / \mathrm{s}$. For the thin $\mathrm{TiO}_{2}$ layer $120 \mathrm{eV}$ and $0.25 \mathrm{~nm} / \mathrm{s}$ were used.

The polymer material on which the BSW multilayers have to be deposited on is a thermoplastic COC (Topas 6013). This material features excellent adhesion to all kinds of evaporated or magnetron sputtered dielectric coatings. So the multilayer coating process could be easily adapted to the polymer requirements in terms of adhesion using a preetching step with the APS source at $80 \mathrm{eV}$ for 60 s pure argon plasma.

\section{Adjusted film densification using ion assisted evaporation}

Considering the film properties, an adjustment of the layer densification was necessary. Highly densified PVD coatings result in huge interface forces on polymer substrates if the coating is thick. Therefore, less densification was applied for the BSW deposition to reduce on the one hand the internal film stress and on the other hand the heat impact on the substrate. Besides that, absorption losses are also lower for less densified coatings as already shown in Figure 2 (right diagram). However, working with the sensor chips in an aqueous environment resulted in a time shift of the resonance peak due to water penetration into the stack. This highly undesired behavior limited temporally 
resolved measurements in biosensing experiments, as the chip itself causes the same shift of the resonance as a biochemical reaction does. Thus, it would falsify the verification results. Moreover, the porous coating started to delaminate after three days storage in water as shown in Figure 4 (left image). However, it has to be noted that these chips should be used as disposable sensors and the coating has to be stable for the time the chip is in use only. Therefore, the densification was optimized on the one hand to respect the delamination problem and on the other hand to keep the film stress and the optical losses as low as possible.

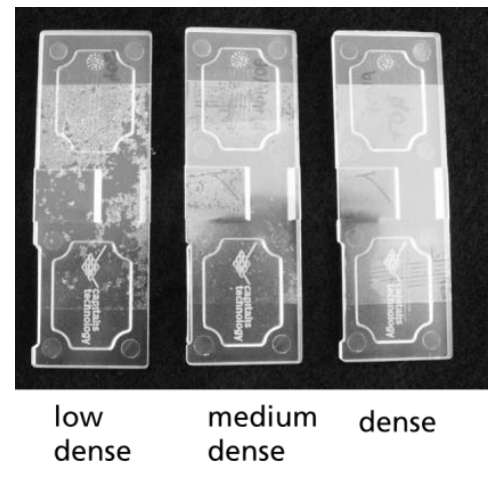

\begin{tabular}{|c|c|c|}
\hline $\begin{array}{c}\text { layer } \\
\text { densification }\end{array}$ & $\begin{array}{c}\text { coating } \\
\text { film stress } \\
\text { [Mpa] }\end{array}$ & $\begin{array}{c}\text { delamination } \\
\text { after water } \\
\text { storage }\end{array}$ \\
\hline low & $\begin{array}{c}-163.20 \\
\text { compressive }\end{array}$ & $>70 \%$ \\
\hline medium & $\begin{array}{c}-277.70 \\
\text { compressive }\end{array}$ & $5-10 \%$ \\
\hline high & $\begin{array}{c}-382.00 \\
\text { compressive }\end{array}$ & $<5 \%$ \\
\hline
\end{tabular}

Figure 4. Coating performance after 3 days water storage for multilayer coatings with different densification (left) and corresponding film stress values (right)

\section{Reliability of the Bloch multilayer stack deposition}

Quartz crystal monitoring was used for the termination of the single layer thicknesses inside the stack. Usually the QCM is calibrated from a recalculation of the layer thicknesses in the stack using a $0^{\circ}$ or $45^{\circ}$ ex situ reflectance measurement. However, in the case of Bloch stacks a deviation in layers where the field intensity is concentrated has a huge effect on the resonance angle measured in total reflectance. Unfortunately, only a minor effect of such variations is observable for the $0^{\circ}$ or $45^{\circ}$ reflectance curve. This introduces a systematic error for the thickness calibration. In addition, layer thickness random errors were in the range of $3 \%$ because of non-perfect coating densification and a resulting vacuum to air shift. The batch to batch variation of four sets, each containing 8 chips, was measured in application conditions with a CCD camera evaluating the angular position of the resonance peak. It was found that the variation between the single batches added to the variation within a batch sums up to approximately $1^{\circ}$ (see Figure 5). This range must be the minimal angular detection range for the analytical instrument that should be assembled later on.

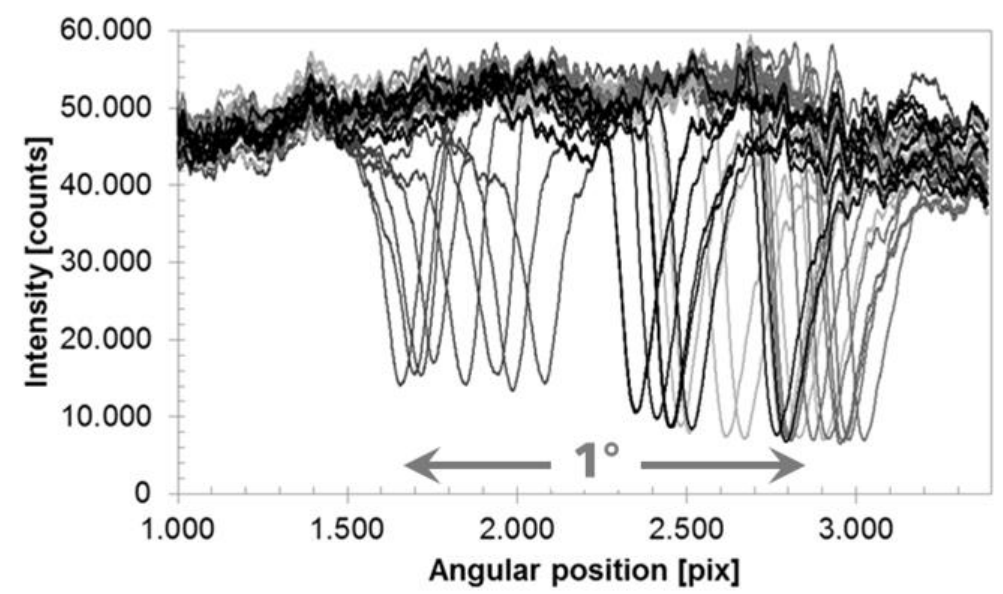

Figure 5. Deviation of the measured resonance peak position for 4 consecutive coating batches each with 8 chips

To minimize systematic thickness errors that originate from insufficient calibration of the quartz crystal monitor coating deposition runs with in-situ broad band optical monitoring (BBM) mounted directly on the rotating substrate holder were performed. This monitoring system is the OptiMon ${ }^{\mathrm{TM}}$ system developed at Fraunhofer IOF previously. 
This BBM applies the recalculation routines from the Optilayer ${ }^{\mathrm{TM}}$ software in a triangular algorithm what allows to determine single layer thicknesses inside the stack with a high precision. It was possible to deposit a Bloch stack with 9 layers including thick layers with $340 \mathrm{~nm}$ and thin layer with $20 \mathrm{~nm}$ from the same material while thick layers exhibited a precision better than $1 \%$ and thin layers better than $1 \mathrm{~nm}$.

\section{Analytical Instrument / Measurement of small biomarker concentrations}

The basic concept for the analytical instrument was to combine label-free and fluorescence mode using the same detector unit. In the label free mode the sensor chip is illuminated from the rear side by TE polarized light of a $670 \mathrm{~nm}$ laser diode. The light is totally reflected at the upper chip surface and directed by some imaging optics to a $\mathrm{CCD}$ array. Here, the resonance peak that turns out to be a dip in reflectance, which appears as a narrow dark line on the CCD image. The position of the resonance is tracked over time, and changes are interpreted as binding or dissociation events at the sensor surface. Thanks to the purposely designed optical system [14], different locations on the chip surface can be analyzed simultaneously. In the fluorescence mode, another light source is used for excitation of a fluorescence dye but the whole detection optics remains the same. The fluorescence evaluation is performed by a wavelength resolved observation of emission intensity changes.

A real label-free measurement of the Angiopoietin 2 a-cancer biomarker was performed by an Aangiopoietin 2 / antibody coupling reaction. Therefore different analytes had to be guided in diminutive amounts to the chip surface what was the task of a complex microfluidic system. The diagram in Figure 6 shows the resonance peak shift over time and illustrates the different steps of such initial experiment that were necessary for verification purposes. Thereby, the decisive peak shift (marked with the arrow) appeared for the case of the specific binding reaction between the antigen (in solution) to its antibody (bound onto the sensor surface). This shift reduces with decreasing Aangiopoietin 2 concentration. So as long as this shift is still distinguishable from the measurement noise an even smaller concentration can be detected what is the final objective e.g. in early cancer diagnosis.
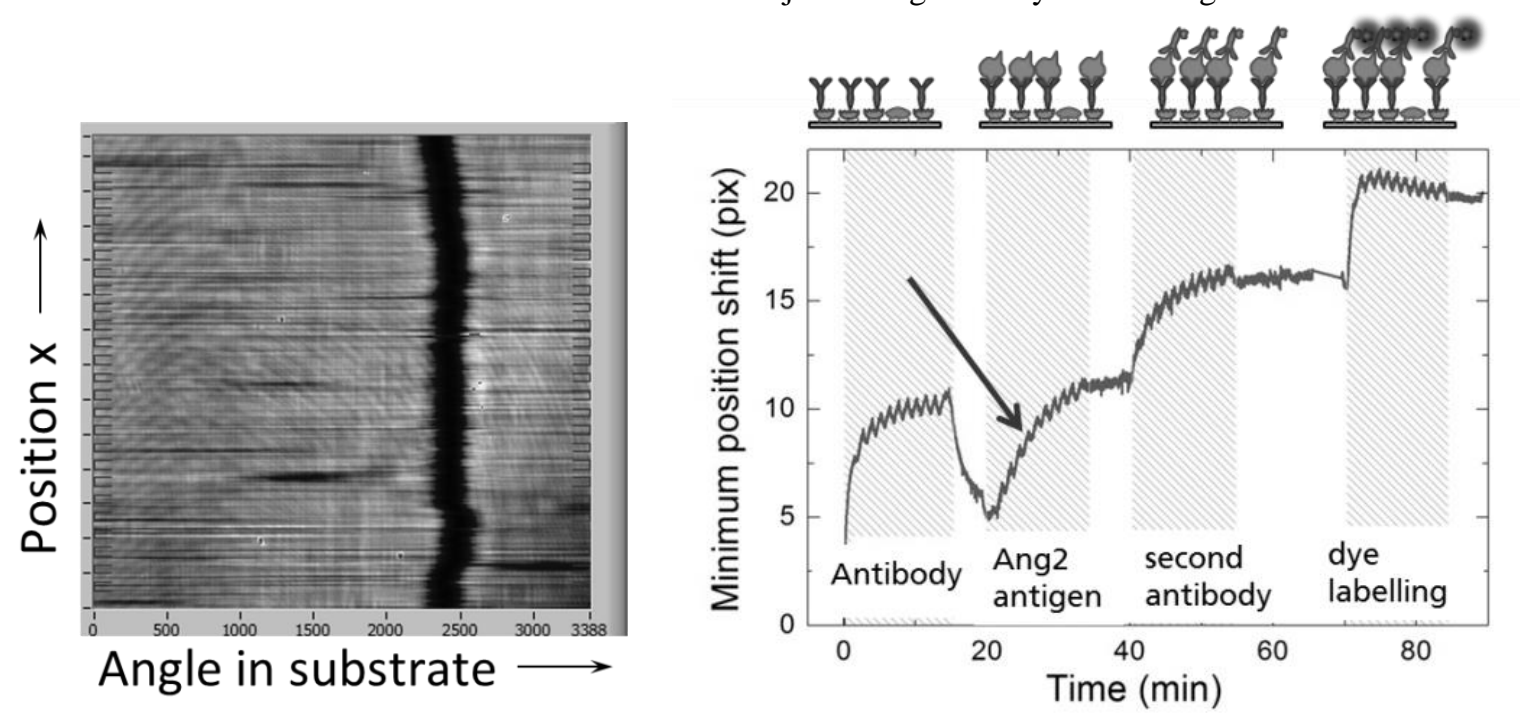

Figure 6. Example of a CCD image obtained in label-free detection modes and measured resonance shift for an Antigen (Angiopoietin2) - Antibody coupling reaction

\section{Conclusion}

A special application for dielectric optical interference coatings was illustrated. In contrast to most optical multilayers where reflectance or transmittance towards air is exploited, stacks for the generation of Bloch Surface Waves (BSW) operate in total internal reflection conditions with the objective of generating an evanescent field on top of the uppermost layer. We were able to demonstrate that the successful deposition of such multilayer stacks on plastic substrates is possible by plasma ion assisted vacuum evaporation (PIAD). Due to the high thickness and refractive index precision on a large area, the flexibility in the densification of the layers and in the adjustment of absorption losses, the PIAD deposition method is well suited for this kind of multilayer coatings. Taking into account 
the size of a typical evaporation box coater, a total of more than 700 polymer chips could be coated within one single deposition run if the substrate holder configuration would be optimal.

By the coating design and the properties of the dielectric layer materials it was possible to tailor the evolving resonance peak in terms of width, depth and dispersion. This adjustability is a clear benefit compared to Surface Plasmon Resonance (SPR) what represents one of the standard optical biosensing methods and works with a thin metal layer whose optical properties restrict the resonance performance. By a proper choice of single layer thicknesses inside a BSW stack e.g. the resonance angle can be tuned for a wide range of operation wavelengths. Therefore, the application of such a sensor also in the UV or IR would be possible. Suitable coating materials with low losses in a wide UV and IR range are state of the art for vacuum evaporation.

\section{Acknowledgments}

This research was funded by the European Commission through the project BILOBA (Grant agreement 318035).

\section{References}

1. R. Lequin, "Enzyme immunoassay (EIA)/enzyme-linked immunosorbent assay (ELISA)", Clin. Chem. 51, $12,2415-8,2005$.

2. J. Homola, "Surface plasmon resonance sensors for detection of chemical and biological species," Chem. Rev. 108, 462-493, 2008.

3. J. Homola, S. S. Yee, and G. Gauglitz, "Surface plasmon resonance sensors: review," Sens. Act. B 54, 3-15, 1999.

4. C. Ciminelli, C. M. Campanella, F. Dell'Olio, C. E. Campanella, and M. N. Armenise, "Label-free optical resonant sensors for biochemical applications," Progr. Quant. Electron. 37, 51-107, 2013.

5. M. Shinn and W. M. Robertson, "Surface plasmon-like sensor based on surface electromagnetic waves in a photonic crystal," Sens. Act. B 105, 360-364, 2005.

6. P. Yeh, A. Yariv, and C.-S. Hong, "Electromagnetic propagation in periodic stratified media. I. General theory," J. Opt. Soc. Am. 67, 423-438, 1977.

7. F. Giorgis, E. Descrovi, C. Summonte, L. Dominici, and F. Michelotti, "Experimental determination of the sensitivity of Bloch Surface Waves based sensors," Opt. Expr. 18, 8087-8093, 2010.

8. V. N. Konopsky, T. Karakouz, E. V. Alieva, C. Vicario, S. K. Sekatskii, and G. Dietler, "Photonic crystal biosensor based on optical surface waves," Sensors 13, 2566-2578, 2013.

9. A. Sinibaldi, R. Rizzo, G. Figliozzi, E. Descrovi, N. Danz, P. Munzert, A. Anopchenko, and F. Michelotti, "A full ellipsometric approach to optical sensing with Bloch surface waves on photonic crystals," Opt. Expr. 21, 23331-23344, 2013.

10. C. Ndiaye, M. Zerrad, A. L. Lereu, R. Roche, P. Dumas, F. Lemarchand, and C. Amra, "Giant optical field enhancement in multi-dielectric stacks by photon scanning tunneling microscopy," Appl. Phys. Lett. 103, $131102,2013$.

11. A. Sinibaldi, N. Danz, E. Descrovi, P. Munzert, U. Schulz, F. Sonntag, L. Dominici, and F. Michelotti, „Direct comparison of the performance of Bloch surface wave and surface plasmon polariton sensors," Sens. Act. B 174, 292-298, 2012.

12. R. Rizzo, N. Danz, F. Michelotti, E. Maillart, A. Anopchenko, C. Wächter, „Optimization of angularly resolved Bloch surface wave biosensors", Opt. Express 22 (19) 23202, 2014.

13. Danz, N., Kick, A., Sonntag, F., Schmieder, S., Höfer, B., Klotzbach, U. and Mertig, M., „Surface plasmon resonance platform technology for multi-parameter analyses on polymer chips," Eng. Life Sci. 11(6) 566572, 2011.

14. Danz, N., Sinibaldi, A., Munzert, P., Anopchenko, A. Förster, E., Schmieder, S., Chandrawati, R., Rizzo, R., Heller, R., Sonntag, F., Mascioletti, A., Rana, S., Schubert, T., Stevens, M.M. and Michelotti, F., „Biosensing platform combining label-free and labelled analysis using Bloch surface waves, “Proc. of SPIE, 9506, 95060V, 2015.

15. H. Raether, Surface Plasmons, edited by G. Höhler, Springer Tracts in Modern Physics Vol. 111 (Springer, Berlin, 1988). 